\title{
Behavior of Plasma Response Field in Detached Plasma
}

\author{
Yoshiro NARUSHIMA, Masahiro KOBAYASHI, Tsuyoshi AKIYAMA, Satoru SAKAKIBARA, \\ Suguru MASUZAKI, Naoko ASHIKAWA and Noriyasu OHNO ${ }^{1)}$ \\ National Institute for Fusion Science, 322-6 Oroshi-cho, Toki 509-5292, Japan \\ 1) Graduate School of Engineering, Nagoya University, Nagoya 464-8603, Japan
}

(Received 25 January 2013 / Accepted 21 March 2013)

\begin{abstract}
Sustaining a detached plasma is important for the reduction of the heat load on a divertor. The externally controlled methods such as gas puffing in the divertor region, etc. have been studied for maintaining detached plasmas. In the recent LHD experiments, a resonant magnetic perturbation (RMP) is utilized to establish the detached plasma, which is one of the candidates to reduce the divertor heat load. The finite plasma response field has been detected when the RMP is imposed, and its behavior is investigated from the viewpoint of the magnetic island dynamics. A parameter of the phase difference, $\Delta \theta$ (defined as the phase difference between the plasma response field and the RMP), changes from antiphase to in-phase while the plasma transits from the attached to the detached states. Finally, when the $\Delta \theta$ reaches at certain critical value, $\Delta \theta_{\text {crit }}$, the plasma enters the detached state. Since this critical value does not have a dependency on plasma beta and collisionality, $\Delta \theta_{\text {crit }}$ seems to be a threshold to establish the detached plasma. From the viewpoint of magnetic island dynamics, the behavior of $\Delta \theta$ approaching in-phase implies the "trend" of island growth. The detached plasma might be established in the case of the tendency for the plasma response field to reinforce the island width.
\end{abstract}

(c) 2013 The Japan Society of Plasma Science and Nuclear Fusion Research

Keywords: LHD, detachment, magnetic island, resonant magnetic perturbation, plasma response field

DOI: $10.1585 /$ pfr.8.1402058

\section{Introduction}

Establishing a method for reducing the divertor heat load is an important issue to realize a nuclear fusion reactor. Although a lot of studies about externally controlled methods have been done to maintain detached plasmas in tokamaks [1,2], a reliable method to obtain a detached plasma has not been found. In recent LHD experiments, a resonant magnetic perturbation (RMP) has been utilized to establish a detached plasma [3], which is one of the candidates to reduce the divertor heat load. When the RMP is imposed on the plasma, a significant plasma response field is observed to modify the magnetic island [4]. The behavior of the plasma response field has been investigated from the viewpoint of magnetic island dynamics [5]. The purpose of this study is to investigate the detailed behavior of the plasma response field and its dependence on plasma parameters for detached plasmas. This article is composed as follows. In the following section, the experimental setup of LHD, its equilibrium and magnetic diagnostics are described. The behavior of the plasma response field in a detached plasma is shown in Sec.3. After a discussion in Sec. 4, we will summarize in the Sec. 5 .

\section{Experiment Setup of LHD}

The LHD is a heliotron-type plasma confinement device with the distinguishing feature being the presence of

author'se-mail: narusima@LHD.nifs.ac.jp a set of continuous helical coils with a poloidal/toroidal winding number $2 / 10$. The helical and poloidal coils used to confine the plasma are superconducting. Major and averaged minor radii of the plasmas studied here are $R=$ $3.9 \mathrm{~m}$ and $a=0.5 \mathrm{~m}$, respectively. The rotational transform $(\iota / 2 \pi)$ profile is monotonically increasing with radius with axis values near $\iota / 2 \pi=0.4$ and an edge value $\iota / 2 \pi \sim 1$ in the vacuum configuration.

\subsection{Resonant magnetic perturbation field and rotational transform profile}

The resonant magnetic perturbation is imposed by the perturbation coil system which had been originally used as a correction coil system to compensate the natural error field [6]. 10 pairs of coils made of normal conductors set at the top and bottom of LHD (Fig. 1) can produce a magnetic field with $m / n=1 / 1$ and/or $2 / 1$ modes (here, $m / n$ are the poloidal/toroidal Fourier mode numbers). In this study, to make the magnetic island with $m / n=1 / 1$, the perturbation field is imposed by RMP coils (shown by black in Fig. 1). In addition, the other RMP coils (shown by gray in Fig. 1) are also used to cancel the toroidal coupling component of $m / n=2 / 1$.

The $\iota / 2 \pi$ profile is modified by the RMP as shown in Fig. 2. Figure 2 (a) shows the rotational profile in the vacuum configuration without an RMP, which shows a monotonic increment from the magnetic axis $(R=3.9 \mathrm{~m})$ to the plasma boundary $(R \sim 3 \mathrm{~m}$ and $\sim 4.6 \mathrm{~m})$. On the other hand, 


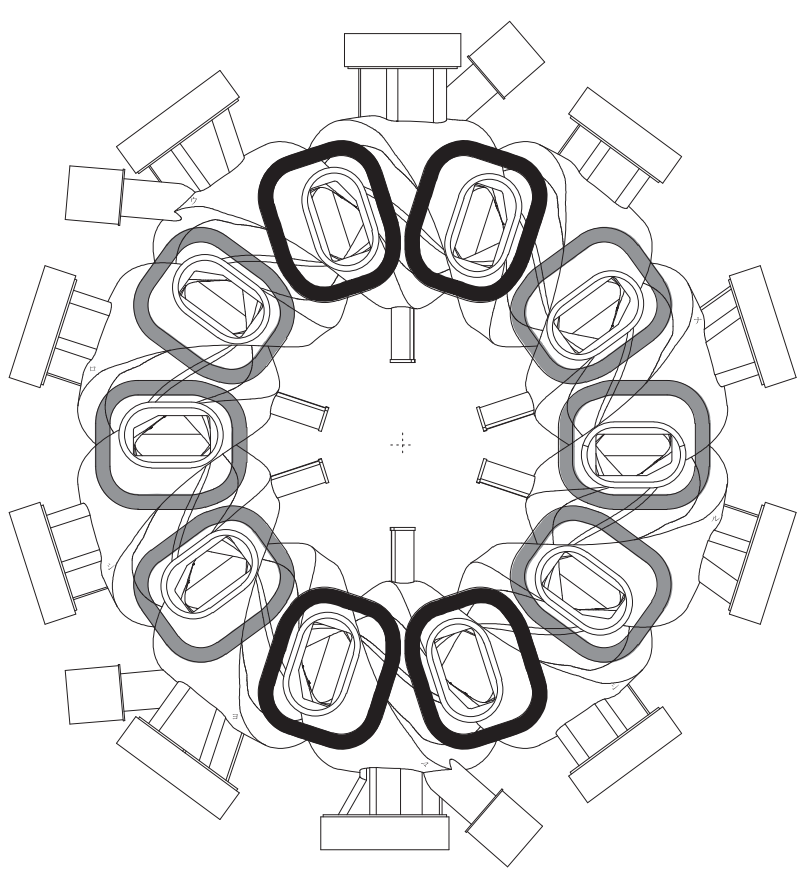

Fig. 1 Top view of vacuum vessel and resonant magnetic perturbation coil system of LHD. Coils for $m / n=1 / 1$ are colored black. Coils for $m / n=2 / 1$ are colored gray.

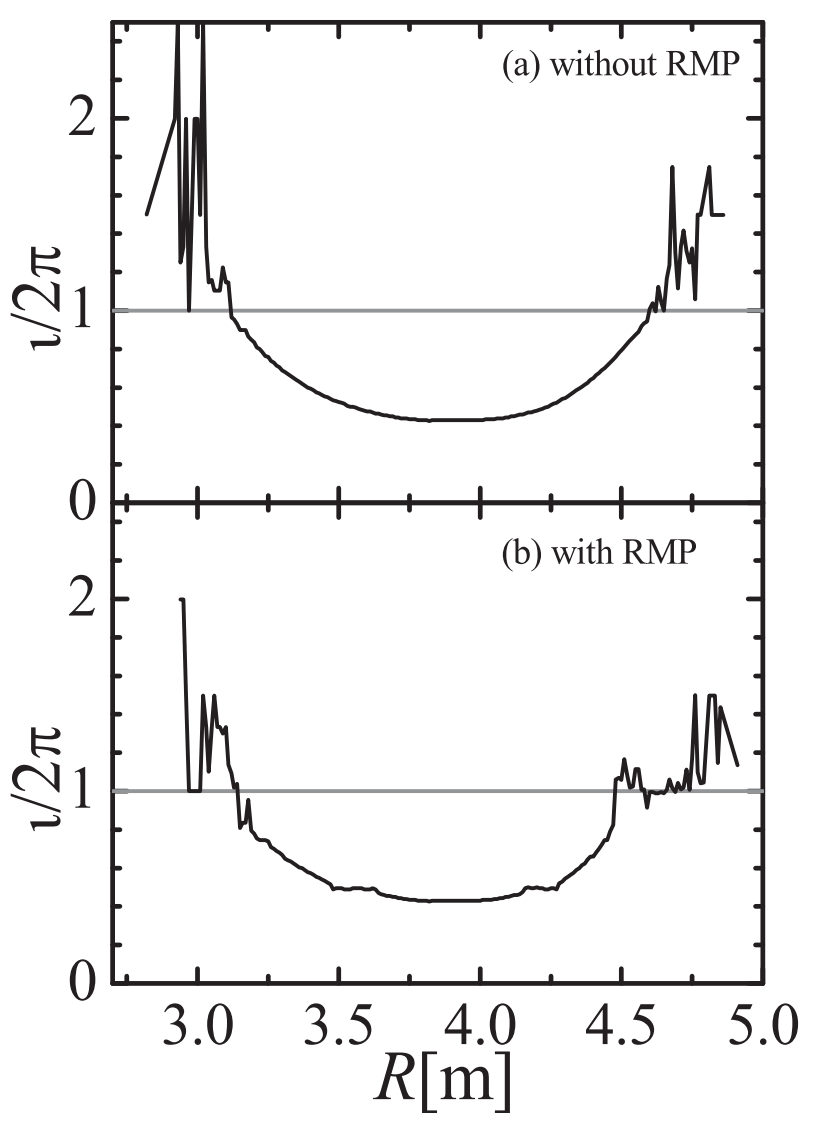

Fig. 2 Major radial profile of rotational transform (a) without and (b) with the RMP. Profile shows local flattening region at $R \sim 4.5 \mathrm{~m}$ in the case an RMP is imposed.

the RMP locally makes the $\iota / 2 \pi$ profile flat at the peripheral region at $R \sim 4.5 \mathrm{~m}$ in Fig. 2 (b), which produces the magnetic island structure.

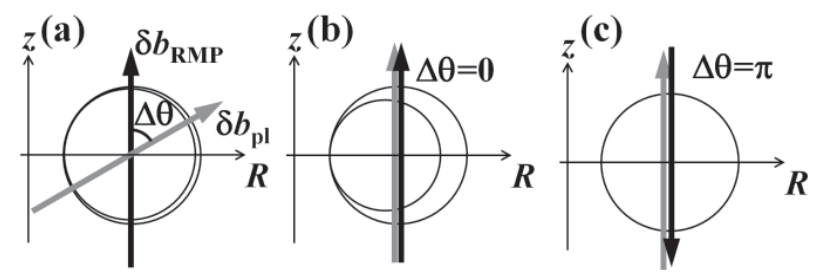

Fig. 3 Explanation drawing of (a) definition of the phase shift $\Delta \theta$, (b) case of in-phase $(\Delta \theta=0)$ leads to island growth, and (c) case of out of phase $(\Delta \theta=\pi)$ leads to island suppression.

\subsection{Measurement of plasma response field}

In the LHD experiments in the presence of plasma, both the growth of the $m / n=1 / 1$ island beyond its vacuum value and disappearance are observed $[4,5]$. When the island grows, the $T_{\mathrm{e}}$ profile shows significant local flattening at the $\iota / 2 \pi=1$ surface. Correspondingly, the magnetic diagnostics show an $m / n=1$ signal that is in-phase with the imposed vacuum magnetic field. Hence, the plasma response produces a larger island-producing radial magnetic field. In the disappeared state, the Thomson profile shows no flattening at the rational surfaces and the magnetic diagnostic shows an $m / n=1$ signal of precisely the same value as the island-inducing vacuum magnetic field but with a $\pi$ phase change. In the this case, the plasma response produces a magnetic field that exactly cancels the resonant vacuum field such that there is no radial component of the resonant magnetic field at the rational surface.

Here, an important parameter, the phase difference $(\Delta \theta)$, should be noted that the $\Delta \theta$ is defined as the poloidal-angle difference between the phase of plasma response field $\left(\delta b_{\mathrm{pl}}\right)$ and the RMP field $\left(\delta b_{\mathrm{RMP}}\right)$ as shown in Fig. $3(\mathrm{a})$. The $\delta b_{\mathrm{pl}}$ and $\delta b_{\mathrm{RMP}}$ indicate the magnetic field amplitude with an $m=1$ Fourier mode. The phase difference, $\Delta \theta$, can be an indicator of the structure of the magnetic island. When the phase difference is in-phase $(\Delta \theta=0)$, as shown in Fig. 3 (b), the magnetic island grows whereas the magnetic island is suppressed when it is out of phase $(\Delta \theta=\pi)$ as shown in Fig. $3(\mathrm{c})$.

\section{Experimental Result}

\subsection{Detached plasmas}

In the previous study in LHD, the $1 / 3 \sim 1 / 10$ reduction of particle flux and heat load on the divertor plate has been observed by means of the divertor-probe measurement [3], which can be thought of as a detachment. While the detached plasma is established in the LHD experiment, the radiation region exists at the stochastic region which is upstream from the divertor leg. In particular, in the case that the magnetic island is imposed, AXUVD and bolometer diagnostics have shown that the radiation region is localized around the X-point of the magnetic island [3,7]. This detachment in LHD can be thought to be a phenomenon similar to an X-MARFE in a tokamak [8], which can be 


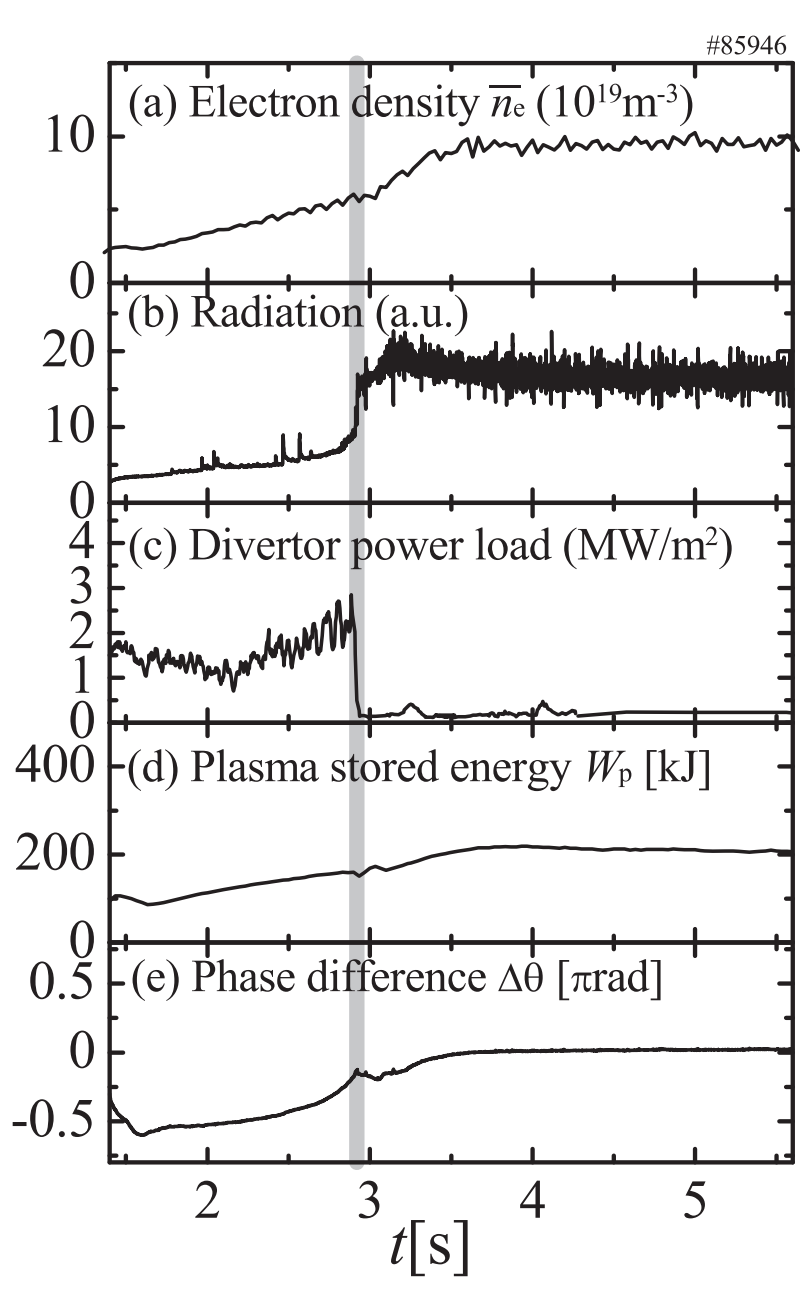

Fig. 4 Time evolution of (a) line averaged electron density, (b) radiation, (c) divertor power load, (d) plasma stored energy, and (e) phase difference. Detached state is established at $t \sim 2.9 \mathrm{~s}$ indicated by gray bold line.

sustained in the stochastic magnetic field at the X-point of the magnetic island.

Figure 4 shows a typical discharge of a detached plasma with an RMP. The plasma transits from an attached to a detached state at $t=2.9 \mathrm{~s}$. At the transition, although the radiation rapidly increases (Fig. 4(b)), the plasma does not experience a radiation collapse. Just after the transition, the signal of the divertor power remains almost zero (Fig. 4 (c)), which means that the divertor heat load can be avoided by means of the establishment of a detached state. A high line-averaged electron density $\left(n_{\mathrm{e}} \sim 10^{20}\left[\mathrm{~m}^{-3}\right]\right)$ is achieved during the detached plasma (Fig. 4(a)). The plasma stored energy increases (Fig. $4(\mathrm{~d})$ ). The phase difference, $\Delta \theta$, gradually moves to the positive direction corresponding to the ion-diamagnetic direction, and the plasmas enter the detached state when $\Delta \theta$ reaches a certain value at $t=2.9 \mathrm{~s}$.

\subsection{Behavior of plasma response field}

Figure 5 summarizes the waveforms of the phase dif-

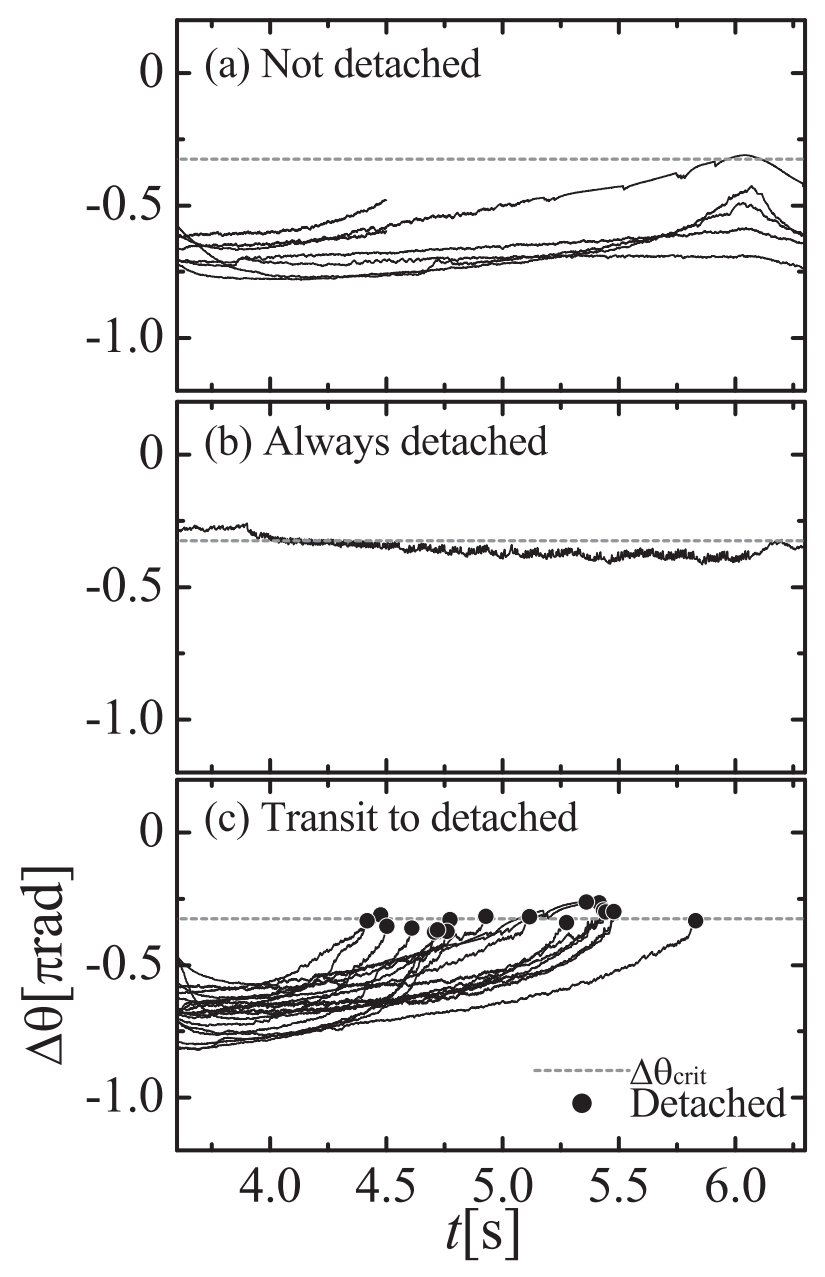

Fig. 5 Time evolution of phase difference $\Delta \theta$ (solid lines) for the cases of (a) not detached, (b) always detached and (c) transition from attached to detached state. Black circles indicate the point when the detached plasmas are established. Gray dashed lines indicate the threshold of $\Delta \theta$ for detachment.

ference, $\Delta \theta$, acquired from multiple discharges. It should be noted that those plasmas have been obtained from the configuration different from that of Fig. 4. The time traces are shown by solid lines. The gray dashed line indicates the critical phase difference $\left(\Delta \theta_{\text {crit }}=-0.32 \pi \mathrm{rad}\right)$ estimated as the average of $\Delta \theta$ when plasmas are in the detached state. In the cases where the plasmas are not detached (Fig. 5 (a)), the $\Delta \theta$ remains less than $\Delta \theta_{\text {crit }}$ whereas the $\Delta \theta$ remains around $\Delta \theta_{\text {crit }}$ when the detached plasma is sustained through the whole term of the discharge (Fig. 5 (b)). On the other hand, in the cases where the plasmas experience a transition to the detached state, $\Delta \theta$ shows dynamical behavior in which the $\Delta \theta$ gradually moves to the positive direction corresponding to ion-diamagnetic direction, and plasmas enter the detached state when the $\Delta \theta$ reaches $\Delta \theta_{\text {crit }}$ as shown in Fig. 5 (c). The values of $\Delta \theta$ for detachment seem to be the same whereas the timings for that are different. 


\section{Discussion}

The experimental fact is that the phase difference, $\Delta \theta$, reaches a critical value $\Delta \theta_{\text {crit }}=-0.32 \pi \mathrm{rad}$ when the plasma transits to a detached state. From the viewpoint of magnetic island dynamics, approaching $\Delta \theta=0$ implies the "trend" of magnetic island growth. In the previous studies $[4,5]$, island growth and suppression can be clearly divided into two regions in the plasma beta $(\beta)$ vs. plasma collisionality $(v)$ space, in which the growth of the magnetic island is realized in higher- $\beta /$ lower- $\nu$ region. However, it should be noted that the previous result $[4,5]$ has been obtained by a magnetic configuration different from this study. Furthermore, the acquired data range is narrower than in $[4,5]$. Therefore, we cannot state that the detachment plasma can be established in higher- $\beta /$ lower- $-v$ region. Nevertheless, the parameter $\Delta \theta$ can show the clear boundary for a detached plasma.

It is worthwhile to investigate the plasma response field depending on $\beta / v$ from the viewpoint of island dynamics. To compare the dispersion of $\Delta \theta, \beta$ and $v$, we summarize them as shown in Fig. 6. Here, the $\beta$ and $v$ are
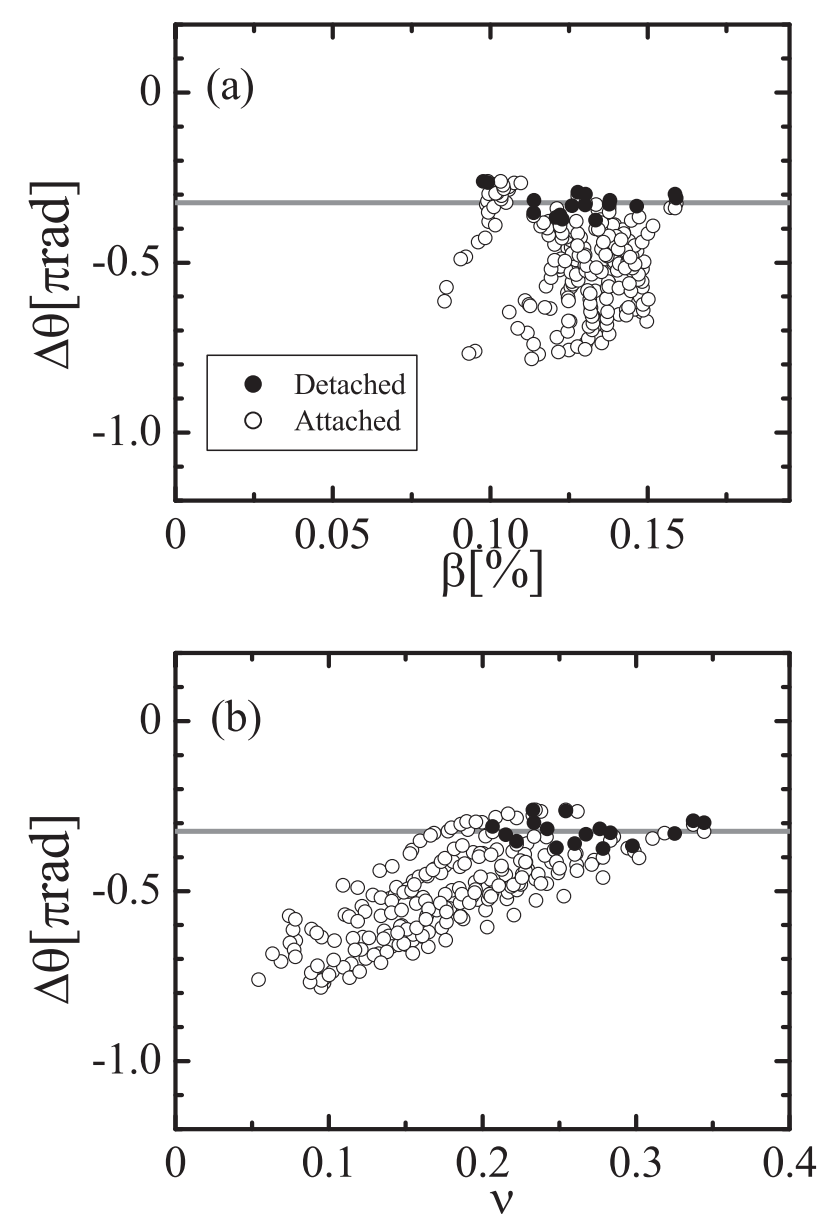

Fig. 6 Relationship between phase difference $\Delta \theta$ and (a) beta and (b) collisionality. Filled and open circles indicate detached and attached state, respectively. Dashed gray line indicates the threshold of $\Delta \theta$ for detachment. calculated values at $\rho=0.9$ (here, $\rho$ is a normalized minor radius). The plasma discharges shown in Fig. 6 are the same as those in Fig. 5 (c). The open and closed circles indicate the data from attached and detached plasmas, respectively. As shown in Fig. 6 (a), about the relationship between $\Delta \theta$ and $\beta$, it seems that there is no correlation between the $\Delta \theta$ and $\beta$. In the cases the plasma is in a detached state (see closed circles), the $\beta$ is widely dispersed in $\beta=0.08-0.17 \%$. Attached plasmas also exist within this $\beta$ range. Namely, it cannot be judged by $\beta$ whether the plasma is in an attached or detached state. As for the collisionality $v$, the correlation between $\Delta \theta$ and $v$ is not weaker than that of the case of $\Delta \theta$ and $\beta$ as shown in Fig. 6 (b). The $\Delta \theta$ approaches in-phase when the $v$ increases. The $v$, however, is also widely dispersed in $v=0.2-0.34$ when the plasma is in the detached state. Noticing the case of detachment (closed circles), the $\beta$ and $v$ are widely dispersed $(\beta=0.08-0.17 \%, v=0.2-0.34)$ whereas the phase difference $\Delta \theta$ is nearly-constant. These experimental observations show that the $\Delta \theta_{\text {crit }}$ seems to be a threshold to establish the detached plasma.

These experimental facts mean that the plasma does not get in the detached state until the magnetic configuration at the peripheral region has a certain structure. That is to say, the detached plasma is established in case of the tendency for the plasma response field to reinforce the island width. Kobayashi [7] has shown that the detachment occurs when the cooling area by carbon radiation is localized around the X-point of the island. For that, the wellstructured magnetic island is required. This statement is consistent with the experimental observation shown in this paper.

\section{Summary}

The behavior of the plasma response field in a detached plasma is studied. In this study, we notice the phase difference $(\Delta \theta)$ defined as the phase difference between the plasma response field and the RMP. When the plasma transits from an attached to a detached state, the phase difference $\Delta \theta$ gradually moves towards in-phase $(\Delta \theta=0)$ to the certain critical value $\Delta \theta_{\text {crit }}$. In multiple discharges with transition, the $\Delta \theta_{\text {crit }}$ is almost the same whereas the plasma beta and collisionality are dispersed. These experimental observations can be interpreted such that the trend of island growth leads to a detached state. That is consistent with the previous study in which a well-structured magnetic island is required to make a detached plasmas [7].

\section{Acknowledgement}

This study was supported by a Grant-in-Aid for Young Scientists (B) (No.22760661) from the Ministry of Education, Culture, Sports, Science and Technology of Japan. This work was supported by NIFS under Contract No.NIFS12ULPP014. 
[1] C.S. Pitcher and P.C. Stangeby, Plasma Phys. Control. Fusion 39, 779 (1997).

[2] O. Gruber, A. Kallenbach, M. Kaufmann, K. Lackner, V. Mertens, J. Neuhauser, F. Ryter, H. Zohm et al., Phys. Rev. Lett. 74, 4217 (1995).

[3] M. Kobayashi, S. Masuzaki, I. Yamada, N. Tamura, Y. Feng, K. Sato, M. Goto, Y. Narushima et al., Phys. Plasmas 17 056111 (2010).

[4] Y. Narushima, K.Y. Watanabe, S. Sakakibara, K. Narihara, I. Yamada, Y. Suzuki, S. Ohdachi, N. Ohyabu et al., Nucl. Fusion 48, 075010 (2008).
[5] Y. Narushima, F. Castejón, S. Sakakibara, K.Y. Watanabe, S. Ohdachi, Y. Suzuki, T. Estrada et al., Nucl. Fusion 51, 083030 (2011).

[6] T. Morisaki, N. Ohyabu, S. Masuzaki, M. Kobayashi, R. Sakamoto, J. Miyazawa, H. Funaba et al., Phys. Plasmas 14, 056113 (2007).

[7] M. Kobayashi, S. Masuzaki, I. Yamada, Y. Narushima, C. Suzuki, N. Tamura et al., 24th IAEA Fusion Energy Conference CN-197 EX/4-4 (2012) San Diego, USA.

[8] B. Lipschultz, J. Nucl. Mater. 15, 145 (1987). 